\title{
Optimum Power Allocation for Beamforming-Based Regenerative Dual-Hop MISO Relay Channels
}

\author{
Hee-Nam Cho, Jin-Woo Lee and Yong-Hwan Lee \\ School of Electrical Engineering and INMC \\ Seoul National University \\ Kwanak P. O. Box 34, Seoul 151-600 Korea \\ e-mail: \{hncho,jinu\}@ttl.snu.ac.kr and ylee@snu.ac.kr
}

\begin{abstract}
We consider a power allocation strategy to maximize the end-to-end capacity of a regenerative dual-hop multiple-input single-output (MISO) relay channel with the use of transmit beamforming. In a dual-hop MISO relay channel, the optimum power allocation can be determined by means of a max-min technique with sum power constraints. The proposed scheme adaptively allocates the transmit power of the source and relay according to the achievable beamforming gain as well as the average channel gain to balance the capacity between the dualhop MISO relay channels. Simulation results show that the proposed scheme can provide noticeable performance improvement over the average signal-to-noise ratio (SNR)-based power allocation scheme in a dual-hop MISO relay channel with the use of the transmit beamforming.
\end{abstract}

\section{INTRODUCTION}

The use of wireless relays has actively been considered in wireless networks due to its potentials for the enhancement of cooperative diversity, capacity, and cell coverage [1]. Relay systems generally employ two types of operating modes, regenerative and non-regenerative relay [2]. Recent researches have focused on the use of regenerative relays, which is less affected from noise enhancement in low signal-to-noise ratio (SNR) environments than the use of non-regenerative relays by employing a decode-and-forward (DF) scheme [3].

In the regenerative relay mode, it is of important concern to allocate the resource (e.g., power and bandwidth) to maximize the end-to-end capacity of a dual-hop relay channel [4]-[7]. When the relays employ a single antenna, the end-to-end capacity can be maximized by balancing the capacity between the first and second hop channels based on the average SNRs of dual-hop channels [4]. Recently, resource allocation for multiple-input multiple-output (MIMO) relay channels have been under consideration due to the ability of MIMO channels, which enables to increase the data rate or decrease the error rate [5]-[7]. In MIMO relay channels, the resource also has been allocated according to the average SNRs of dual-hop MIMO relay channels with various transmission strategies (e.g., dirty-paper coding and distributed space-time coding) [5]. However, the resource allocation for the relays with the use of transmit beamforming has not been reported.

In this paper, we consider a power allocation for regenerative dual-hop multiple-input single-output (MISO) relay systems with the use of transmit beamforming in spatially-correlated channel environments. We optimize the transmit power to maximize the end-to-end capacity considering the achievable beamforming gain as well as the average channel gain between dual-hop MISO relay channels. We also consider the use of short-term channel state information (CSI) and long-term CSI for generating the beamforming vector at the source and relay [8], [9]. The performance of the proposed scheme is analyzed and verified by computer simulation. The analytic and numerical results show that the proposed scheme significantly outperforms the average SNR-based power allocation scheme in beamforming-based regenerative dual-hop relay channels.

The remainder of this paper is organized as follows. Section II describes a correlated dual-hop MISO relay channel with the use of the transmit beamforming in consideration. Section III proposes an optimum power allocation strategy that maximizes the end-to-end capacity of beamforming-based dual-hop MISO relay channels. Section IV verifies the performance of the proposed scheme by computer simulation. Finally, conclusions are given in Section V.

\section{SYSTEM MODEL}

We consider a regenerative dual-hop MISO relay system as shown in Fig. 1, where the source transmits the signal using $M_{1}$ antennas to the relay by means of transmit beamforming, the relay receives it using a single antenna and re-transmits it using $M_{2}$ antennas to the destination by means of transmit beamforming, the destination receives it using a single antenna. We assume that the first and second hop channel equally share the available channel bandwidth, and that the total sum power of the source and relay is $p_{0}$. We also assume that the direct link between the source and the destination is unavailable due to large path loss.

Let $s_{i}, \mathbf{w}_{i}$, and $\mathbf{h}_{i}$ be the transmit signal, $\left(M_{i} \times 1\right)$ transmit beamforming vector, and $\left(1 \times M_{i}\right)$ channel vector of the $i$-th hop channel, respectively. Then, the received signal through the $i$-th hop channel can be represented as

$$
y_{i}=\sqrt{\gamma_{i}} \mathbf{h}_{i} \mathbf{w}_{i} s_{i}+z_{i}
$$

where $z_{i}$ denotes additive noise of the $i$-th hop channel, which is a zero-mean complex Gaussian random variable with variance $\sigma_{i}^{2}$, and $\gamma_{i}\left(\triangleq p_{i} d_{i}^{-\tau}\right)$ denotes the large-scale fading 


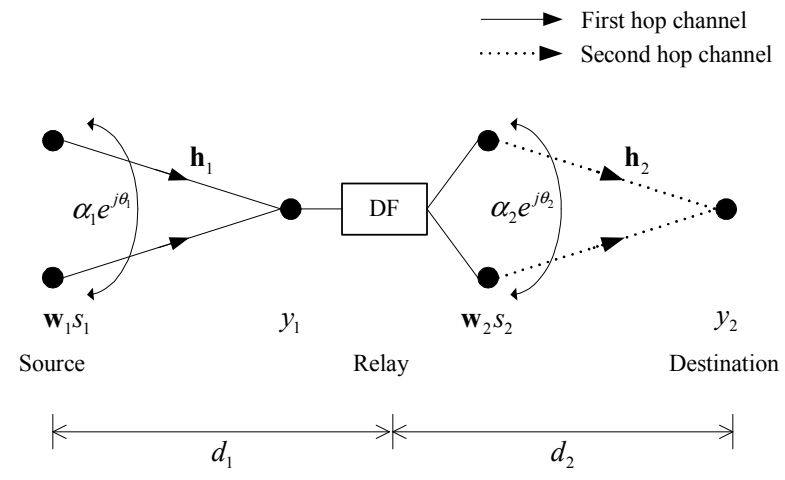

Fig. 1. Modeling of a dual-hop MISO relay systems.

coefficient of the $i$-th hop channel. Here, $p_{i}$ is the transmit power, $d_{i}$ is the propagation distance, and $\tau$ is the path loss exponent.

Assuming that each hop channel experiences spatiallycorrelated Rayleigh fading, the channel vector $\mathbf{h}_{i}$ can be generated using an independent and identically distributed (i.i.d) Rayleigh channel vector $\tilde{\mathbf{h}}_{i}$ by [12]

$$
\mathbf{h}_{i}=\tilde{\mathbf{h}}_{i} \mathbf{R}_{i}^{1 / 2}
$$

where $\mathbf{R}_{i}^{1 / 2}$ denotes the square root of the channel covariance matrix $\mathbf{R}_{i}$ defined by [13]

$$
\begin{aligned}
\mathbf{R}_{i} & =E\left\{\mathbf{h}_{i}^{*} \mathbf{h}_{i}\right\} \\
& =\left[\begin{array}{cccc}
1 & \rho_{i} & \cdots & \rho_{i}^{M_{i}-1} \\
\rho_{i}^{*} & 1 & \cdots & \rho_{i}^{M_{i}-2} \\
\vdots & \vdots & \ddots & \vdots \\
\rho_{i}^{*\left(M_{i}-1\right)} & \rho_{i}^{*\left(M_{i}-2\right)} & \cdots & 1
\end{array}\right] .
\end{aligned}
$$

Here, the superscript $*$ denotes conjugate transpose, $E\{\mathbf{x}\}$ denotes the expectation of $\mathbf{x}$, and $\rho_{i}\left(\triangleq \alpha_{i} e^{j \theta_{i}}\right)$ denotes the transmit correlation coefficient between adjacent antennas, where $\alpha_{i} \quad\left(0 \leq \alpha_{i} \leq 1\right)$ and $\theta_{i} \quad\left(0 \leq \theta_{i} \leq 2 \pi\right)$ denote its amplitude and phase, respectively. Since $\mathbf{R}_{i}$ is a positive definite Hermitian matrix, it can be decomposed as [13]

$$
\mathbf{R}_{i}=\mathbf{U}_{i} \boldsymbol{\Sigma}_{i} \mathbf{U}_{i}^{*}
$$

where $\mathbf{U}_{i}=\left[\begin{array}{lll}\mathbf{u}_{i, 1} & \cdots & \mathbf{u}_{i, M_{i}}\end{array}\right]$ is an $\left(M_{i} \times M_{i}\right)$-dimensional unitary matrix whose columns are the normalized eigenvectors of $\mathbf{R}_{i}$ and $\boldsymbol{\Sigma}_{i}$ is an $\left(M_{i} \times M_{i}\right)$-dimensional diagonal matrix whose diagonal elements $\left\{\lambda_{i, 1}, \ldots, \lambda_{i, M_{i}}\right\}$ are descending ordered non-negative real values, i.e., $\lambda_{i, 1} \geq \ldots \geq \lambda_{i, M_{i}} \geq 0$.

\section{Proposed Power AlLocAtion Scheme}

In this section, we consider power allocation of a regenerative dual-hop MISO relay channel with the use of transmit beamforming. We first derive the capacity of each hop channel with the use of beamforming, and determine the transmit power to maximize the end-to-end capacity of a dualhop MISO relay channel by means of a max-min technique [14]. Finally, the optimum strategy is applied to regenerative dual-hop MISO relay systems with the use of two beamforming schemes, coherent beamforming based on short-term CSI and eigen-beamforming based on long-term CSI.

\section{A. Proposed Power Allocation Scheme}

When the source and the relay transmit the signal with a beamforming vector $\mathbf{w}_{i}$ for $i=1,2$, the capacity of the $i$-th hop channel can be represented as [13]

$$
C_{i}\left(p_{i}\right)=E\left\{\log _{2}\left(1+\frac{\gamma_{i}}{\sigma_{i}^{2}}\left|\mathbf{h}_{i} \mathbf{w}_{i}\right|^{2}\right)\right\}
$$

where $p_{i}$ is the transmit power of the $i$-th hop channel.

Since the end-to-end capacity of a regenerative dual-hop MISO relay channel is determined by the minimum capacity between dual-hop channels, i.e., $C=\min \left(C_{1}\left(p_{1}\right), C_{2}\left(p_{2}\right)\right)$ [1], it is desirable to distribute the total power $p_{0}$ to maximize the minimum capacity between dual-hop channels. Thus, the optimum power allocation problem for a regenerative dual-hop MISO relay channel can be formulated as

$$
C=\max _{\left(p_{1}, p_{2}\right)} \min \left\{C_{1}\left(p_{1}\right), C_{2}\left(p_{2}\right)\right\} \text { subject to } p_{1}+p_{2}=p_{0} .
$$

By means of a max-min technique [14], it can be shown that the end-to-end capacity of a regenerative dual-hop MISO relay channel can be maximized by making

$$
C_{1}\left(p_{1}^{\mathrm{opt}}\right)=C_{2}\left(p_{2}^{\mathrm{opt}}\right)
$$

Utilizing the relationship (7) between the first and second hop channel, we can derive the optimum power allocation for a dual-hop MISO relay channel with the use of transmit beamforming. It can be shown from Jensen's inequality [13] that

$$
\begin{aligned}
C_{i}\left(p_{i}\right) & \leq \log _{2}\left(1+\frac{\gamma_{i}}{\sigma_{i}^{2}} E\left\{\left|\mathbf{h}_{i} \mathbf{w}_{i}\right|^{2}\right\}\right) \\
& =\log _{2}\left(1+\frac{\gamma_{i}}{\sigma_{i}^{2}} \kappa_{i}\right)
\end{aligned}
$$

where $\kappa_{i}\left(\triangleq E\left\{\left|\mathbf{h}_{i} \mathbf{w}_{i}\right|^{2}\right\}\right)$ denotes the beamforming gain of the $i$-th hop channel. Thus, it can be seen that the condition satisfying (7) is given by

$$
\frac{\gamma_{1}}{\sigma_{1}^{2}} \kappa_{1}=\frac{\gamma_{2}}{\sigma_{2}^{2}} \kappa_{2}
$$


Since $\gamma_{i}=p_{i} d_{i}^{-\tau}$, (9) can be rewritten as

$$
p_{2}=\frac{\kappa_{1}}{\kappa_{2}}\left(\frac{d_{1}^{-\tau} / \sigma_{1}^{2}}{d_{2}^{-\tau} / \sigma_{2}^{2}}\right) p_{1}
$$

By inserting (10) into the sum power constraint in (6), it can be shown that the transmit power of a dual-hop MISO relay channel with the use of transmit beamforming can be optimally determined as

$$
\left(p_{1}^{\mathrm{opt}}, p_{2}^{\mathrm{opt}}\right)=\left(\frac{1}{1+\frac{\kappa_{1}}{\kappa_{2}}\left(\frac{d_{1}^{-\tau} / \sigma_{1}^{2}}{d_{2}^{-\tau} / \sigma_{2}^{2}}\right)} p_{0}, \frac{1}{1+\frac{\kappa_{2}}{\kappa_{1}}\left(\frac{d_{2}^{-\tau} / \sigma_{2}^{2}}{d_{1}^{-\tau} / \sigma_{1}^{2}}\right)} p_{0}\right) .
$$

It can be seen that the transmit power $\left(p_{1}^{\text {opt }}, p_{2}^{\text {opt }}\right)$ should be determined by the achievable beamforming gain $\kappa_{i}$ as well as the average channel gain $d_{i}^{-\tau} / \sigma_{i}^{2}$. In fact, $p_{i}^{\text {opt }}$ is inversely proportional to the average channel gain and the beamforming gain of the $i$-th hop channel. Thus, when the average channel gain and beamforming gain of the first hop channel are larger than that of the second hop channel, it needs to decrease $p_{1}^{\text {opt }}$ and increase $p_{2}^{\text {opt }}$ to balance the capacity of the first and the second hop channel, and vice versa.

\section{B. Application to Beamforming Schemes}

We consider the application of the proposed power allocation scheme to two beamforming schemes.

\section{1) Coherent Beamforming}

When the signal is transmitted by means of coherent beamforming with beamforming weight [8]

$$
\mathbf{w}_{i, \mathrm{CB}}=\mathbf{h}_{i}^{*} /\left\|\mathbf{h}_{i}\right\| \text { for } i=1,2
$$

it can easily be shown that the end-to-end capacity can be maximized by allocating the transmit power as

$$
\left(p_{1, \mathrm{CB}}^{\mathrm{opt}}, p_{2, \mathrm{CB}}^{\mathrm{opt}}\right)=\left(\frac{1}{1+\frac{M_{1}}{M_{2}}\left(\frac{d_{1}^{-\tau} / \sigma_{1}^{2}}{d_{2}^{-\tau} / \sigma_{2}^{2}}\right)} p_{0}, \frac{1}{1+\frac{M_{2}}{M_{1}}\left(\frac{d_{2}^{-\tau} / \sigma_{2}^{2}}{d_{1}^{-\tau} / \sigma_{1}^{2}}\right)} p_{0}\right) .
$$

It can be seen that the transmit power should be determined according to the number of transmit antennas $M_{i}$ as well as the average channel gain $d_{i}^{-\tau} / \sigma_{i}^{2}$ since the coherent beamforming gain $\kappa_{i, \mathrm{CB}}\left(\triangleq E\left\{\left|\mathbf{h}_{i} \mathbf{w}_{i, \mathrm{CB}}\right|^{2}\right\}\right)$ of the $i$-th hop channel equals to $M_{i}$.

\section{2) Eigen Beamforming}

When the signal is transmitted by means of eigenbeamforming, the beamforming weight can be determined by the principal eigenvector of the transmit correlation matrix of each hop MISO channel as [9]

$$
\mathbf{w}_{i, \mathrm{~EB}}=\mathbf{u}_{i, \max } \text { for } i=1,2 .
$$

where $\mathbf{u}_{i, \max }$ denotes the principal eigenvector corresponding to $\lambda_{i, 1}$. It can be shown that the eigen-beamforming gain $\kappa_{i, \mathrm{~EB}}$ of the $i$-th hop channel can be represented as

$$
\begin{aligned}
\kappa_{i, \mathrm{~EB}} & =E\left\{\left|\mathbf{h}_{i} \mathbf{u}_{i, \max }\right|^{2}\right\} \\
& =E\left\{\mathbf{h}_{i} \mathbf{u}_{i, \max } \mathbf{u}_{i, \max }^{*} \mathbf{h}_{i}^{*}\right\} .
\end{aligned}
$$

Since $\mathbf{h}_{i}=\tilde{\mathbf{h}}_{i} \mathbf{R}_{i}^{1 / 2}$ and $E\left\{\tilde{\mathbf{h}}_{i} \mathbf{A} \tilde{\mathbf{h}}_{i}^{*}\right\}=\operatorname{tr}(\mathbf{A})$ [13], (15) can be represented as

$$
\begin{aligned}
\kappa_{i, \mathrm{~EB}} & =E\left\{\tilde{\mathbf{h}}_{i} \mathbf{R}_{i}^{1 / 2} \mathbf{u}_{i, \max } \mathbf{u}_{i, \max }^{*} \mathbf{R}_{i}^{* / 2} \tilde{\mathbf{h}}_{i}^{*}\right\} \\
& =E\left\{\tilde{\mathbf{h}}_{i} \mathbf{R}_{i} \mathbf{u}_{i, \max } \mathbf{u}_{i, \max }^{*} \tilde{\mathbf{h}}_{i}^{*}\right\} \\
& =\operatorname{tr}\left(\mathbf{R}_{i} \mathbf{u}_{i, \max } \mathbf{u}_{i, \max }^{*}\right) \\
& =\lambda_{i, \max } .
\end{aligned}
$$

Thus, the optimum power allocation that maximizes the end-toend capacity can be determined as

$$
\left(p_{1, \mathrm{BB}}^{\mathrm{opt}}, p_{2, \mathrm{~EB}}^{\mathrm{opt}}\right)=\left(\frac{1}{1+\frac{\lambda_{1, \text { max }}}{\lambda_{2, \text { max }}}\left(\frac{d_{1}^{-\tau} / \sigma_{1}^{2}}{d_{2}^{-\tau} / \sigma_{2}^{2}}\right)} p_{0}, \frac{1}{1+\frac{\lambda_{2, \text { max }}}{\lambda_{1, \text { max }}}\left(\frac{d_{2}^{-\tau} / \sigma_{2}^{2}}{d_{1}^{-\tau} / \sigma_{1}^{2}}\right)} p_{0}\right)
$$

It can be seen that when the eigen-beamforming is employed, the transmit power needs to be allocated according to the principal eigen-value $\lambda_{i, \max }$ of transmit correlation matrix and the average channel gain $d_{i}^{-\tau} / \sigma_{i}^{2}$. As a special case, when two transmit antennas are employed (i.e, $M_{i}=2$ ), the optimum power allocation can be represented as

$$
\left(p_{1, \mathrm{BB}}^{\mathrm{opt}}, p_{2, \mathrm{BB}}^{\mathrm{opt}}\right)=\left(\frac{1}{1+\frac{1+\alpha_{1}}{1+\alpha_{2}}\left(\frac{d_{1}^{-\tau} / \sigma_{1}^{2}}{d_{2}^{-\tau} / \sigma_{2}^{2}}\right)} p_{0}, \frac{1}{1+\frac{1+\alpha_{2}}{1+\alpha_{1}}\left(\frac{d_{2}^{-\tau} / \sigma_{2}^{2}}{d_{1}^{-\tau} / \sigma_{1}^{2}}\right)} p_{0}\right)
$$

since $\lambda_{i, \max }=1+\alpha_{i}$ for $M_{i}=2$ [10]. It can be seen that the transmit power can simply be determined by amplitude $\alpha_{i}$ of the transmit correlation coefficient and the average channel gain $d_{i}^{-\tau} / \sigma_{i}^{2}$ between dual-hop channels.

\section{SIMULATION RESULTS}

The performance of the proposed power allocation scheme is verified by computer simulation. For comparison, the performance of the average SNR-based power allocation scheme [5] is also considered, where the total power is 
distributed according to only the average channel gain between dual-hop channels. The common simulation parameters are summarized in Table I.

Fig. 2 depicts the allocated power of the first hop channel with use of the coherent beamforming according to the number of source antennas $M_{1}$ when $d_{1}=0.7 \mathrm{~km}, d_{2}=0.3 \mathrm{~km}$, $\alpha_{1}=0.9, \alpha_{1}=0.5, M_{2}=2$, and $\gamma_{0}=10 \mathrm{~dB}$. It can be seen that the proposed scheme distributes less power to the first hop channel than the average SNR-based scheme. This is mainly because the beamforming gain of the first hop channel is larger than that of the second hop channel since $M_{1}>M_{2}$. When $M_{1}=2$, the transmit power of the proposed scheme equals to that of the average SNR-based power allocation scheme since $\kappa_{i, \text { Prop }}>\kappa_{i, \mathrm{SNR}}$.

Fig. 3 depicts the end-to-end capacity of the proposed scheme with use of the coherent beamforming according to $\gamma_{0}$ when $d_{1}=0.7 \mathrm{~km}, d_{2}=0.3 \mathrm{~km}, \alpha_{1}=0.9, \alpha_{2}=0.5$, $M_{1}=4$, and $M_{2}=2$. It can be seen that the proposed scheme noticeably outperforms the average SNR-based scheme. This is mainly because the proposed scheme adaptively distributes the transmit power according to the number of transmit antennas as well as the average channel gain between dual-hop MISO channels. It can be also seen that the analytic results are slightly larger than the simulation results due to Jensen's inequality in (8).

Fig. 4 depicts the allocated transmit power of the first hop channel according to $\Delta \alpha=\left|\alpha_{1}-\alpha_{2}\right|$ when the proposed scheme is applied to use of the eigen-beamforming with $d_{1}=0.7 \mathrm{~km}, d_{2}=0.3 \mathrm{~km}, \alpha_{1}=0, M_{1}=M_{2}=2$, and $\gamma_{0}=10 \mathrm{~dB}$. It can be seen that the proposed scheme distributes more power to the first hop channel than the average SNR-based scheme. This is mainly because the beamforming gain of the first hop channel is smaller than that of the second hop channel since $\alpha_{1}<\alpha_{2}$.

Fig. 5 depicts the end-to-end capacity of the proposed scheme with the eigen-beamforming according to $\gamma_{0}$ for $d_{1}=0.7 \mathrm{~km}, d_{2}=0.3 \mathrm{~km}, \alpha_{1}=0.9, \alpha_{2}=0.5, \quad$ and $M_{1}=M_{2}=2$. It can be seen that the power allocation based on the channel correlation and the average channel gain outperforms one based on the average channel gain.

\section{CONCLUSION}

We have considered a power allocation strategy that maximizes the end-to-end capacity of a regenerative dual-hop MISO relay channels with the use of transmit beamforming. The proposed scheme can adaptively allocate the transmit power according to the beamforming gain as well as the average channel gain between the dual-hop MISO relay channels to balance the capacity of each hop channel. The analytic and simulation results have shown that the proposed scheme can provide noticeable performance improvement over the average SNR-based scheme.
Table I. Simulation parameter

\begin{tabular}{ll}
\hline \multicolumn{1}{c}{ PARAMETERS } & \multicolumn{1}{c}{ VALUES } \\
\hline Relay protocol mode & Regenerative relay with DF scheme \\
Number of hops & 2 \\
Cell radius & $1 \mathrm{~km}$ \\
Source antenna configuration & 2 transmit antennas \\
Relay antenna configuration & 2 transmit, 1 receive antenna \\
Destination antenna configuration & 1 receive antenna \\
Sum power & 1 \\
Fading channel & Spatially-correlated Rayleigh fading \\
Path loss exponent & 4 \\
Link adaptation & Ideal (i.e., using the Shannon's \\
\hline \hline
\end{tabular}

\section{ACKNOWLEDGEMENT}

This work was supported by the Korea Science and Engineering Foundation (KOSEF) grant funded by the Korea government (MEST) (R01-2008-000-21098-0).

\section{REFERENCES}

[1] T. M. Cover and A. A. El Gamal. "Capacity theorems for the relay channel," IEEE Trans. Inf. Theory, vol. 25, no. 5, pp. 572-584, Sept. 1979.

[2] J. N. Laneman, D. N. C. Tse, and G. W. Wornell, "Cooperative diversity in wireless networks: efficient protocols and outage behavior," IEEE Trans. Inf. Theory, vol. 50, no. 12, pp. 3062-3080, Dec. 2004.

[3] B. Wang, J. Zhang, and A. Host-Madsen, "On the capacity of MIMO relay channels," IEEE Trans. Inf. Theory, vol. 51, no. 1, pp. 29-43, Jan. 2005.

[4] A. Host-Madsen and J. Zhang, "Capacity bounds and power allocation for the wireless relay channel," IEEE Trans. Inf. Theory, vol. 51, no. 6, pp. 2020-2040, June 2005.

[5] M. Dohler, A. Gklias, and H. Aghvami, "2-hop distributed MIMO communication system," IEEE Electron. Lett., vol. 39, no. 18, pp. 13501351, Sept. 2003.

[6] Y. Liang and V. V. Veeravalli, "Gaussian orthogonal relay channels: optimal resource allocation and capacity," IEEE Trans. Inf. Theory, vol. 51, no. 9, pp. 3284-3289, Sept. 2005.

[7] Y. Liang, V. V. Veeravalli, and H. V. Poor, "Resource allocation for wireless fading relay channels: max-min solution," IEEE Trans. Inf. Theory, vol. 53, no. 10, pp. 3432-3453, Oct. 2007.

[8] J. Choi, "Pilot channel-aided techniques to compute the beamforming vector for CDMA systems with antenna array," IEEE Trans. Veh. Technol., vol. 49 , no. 5, pp. $1760-1775$, Sept. 2000.

[9] C. Brunner, W. Utschich, and J. A. Nossek, "Exploiting the short-term and long-term channel properties in space and time: eigen beamforming concepts for the BS in WCDMA," Eur. Trans. Telecomm., vol. 12, no. 5, pp. 365-378, Aug. 2001.

[10] G. Caire and S. Shamai, "On the achievable throughput of a multiantenna Gaussian broadcast channel," IEEE Trans. Inf. Theory, vol. 49, no. 7, pp. 1691-1706, July 2003.

[11] A. Goldsmith, S. Ali Jafar, N. Jindal, and S. Vishwanath, "Fundamental capacity of MIMO channels," IEEE J. Sel. Areas Commum., vol. 21, no 5, pp. 684-702, June 1998.

[12] A. Paulraj, R. Nabar, and D. Gore, Introduction to Space-Time Wireless Communications, Cambridge, 2003.

[13] D. Tse and P. Vishwanath, Fundamentals of Wireless Communication, Cambridge University Press, 2005.

[14] V. Poor, An Introduction to Signal Detection and Estimation, Springer, 1994. 


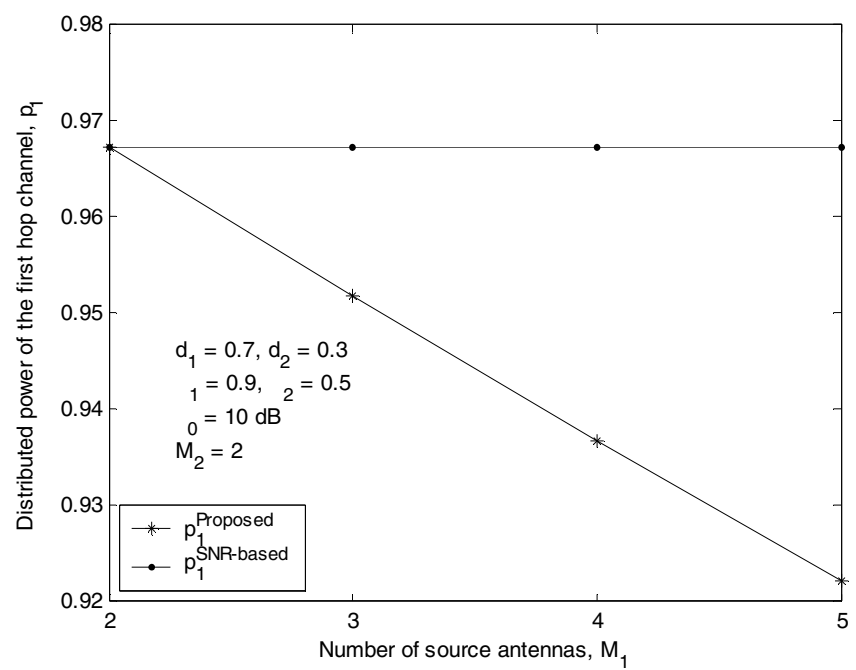

Fig. 2. Distributed power of the first hop channel with the use of coherent beamforming according to $M_{1}$.

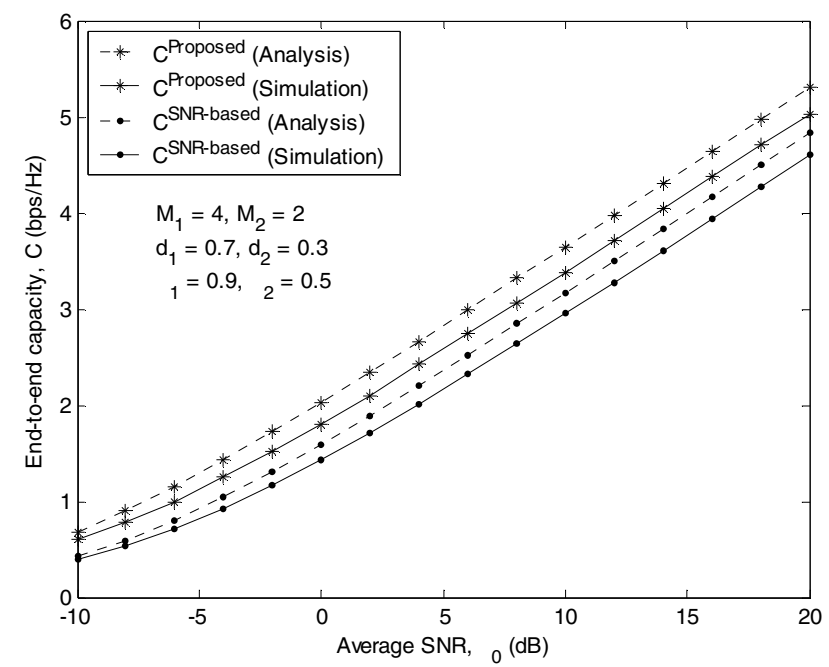

Fig. 3. End-to-end capacity of dual-hop relay channels with the use of coherent beamforming according to $\gamma_{0}$.

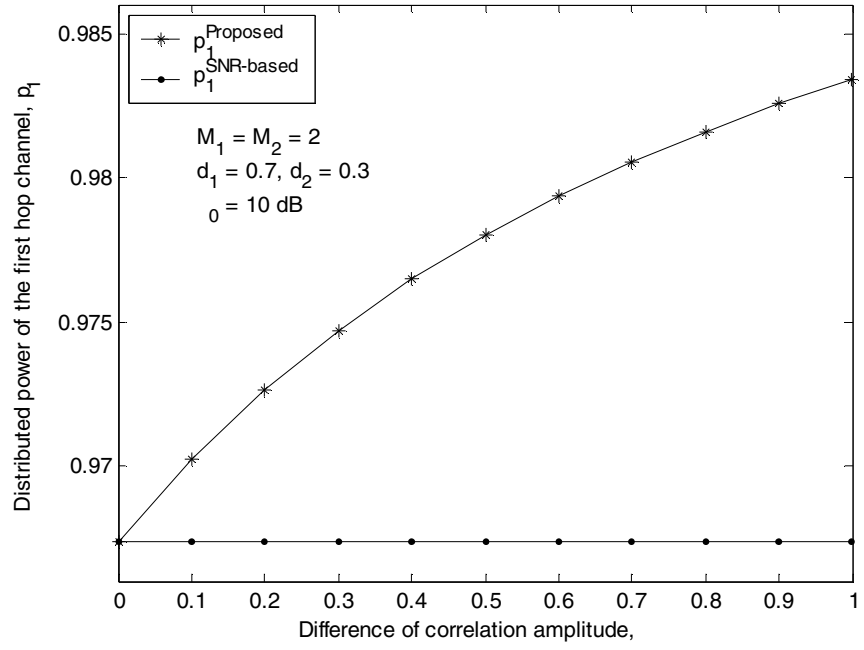

Fig. 4. Distributed power of the first hop channel with the use of eigenbeamforming according to $\Delta \alpha$.

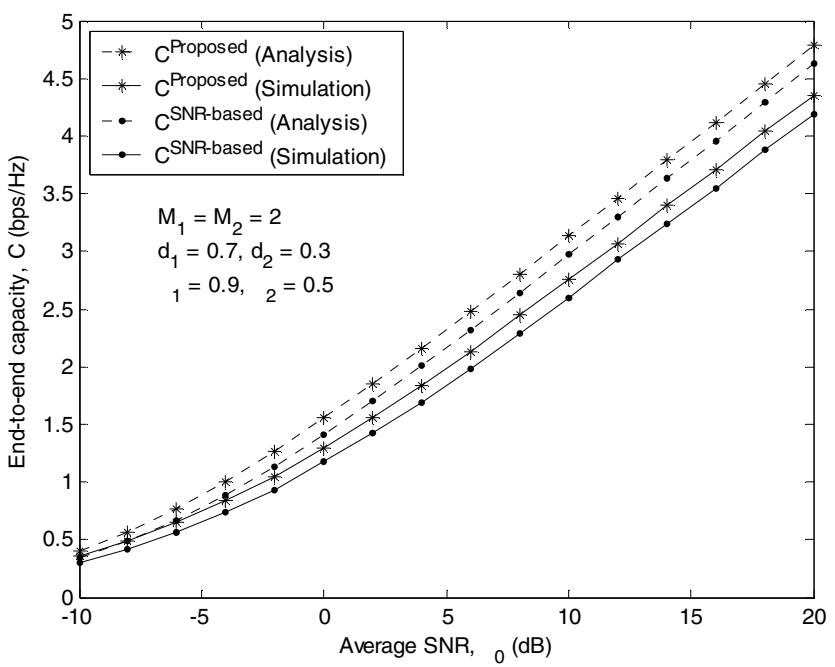

Fig. 5. End-to-end capacity of dual-hop relay channels with the use of eigenbeamforming according to $\gamma_{0}$. 